Ann. Sci. forest., I976, 33 (2), 6I-86.

\title{
PROBLEMES D'INTERDATATION CHEZ LE PIN SYLVESTRE ET INFLUENCE DU CLIMAT SUR LA STRUCTURE DE SES ACGROISSEMENTS ANNUELS
}

\author{
Françoise HUBER \\ Station de Recherches sur la Qualité des Bois, \\ Centre national de Recherches forestières, I. N.R. A., \\ Champenoux, 54280 Seichamps
}

\section{RÉSUMÉ}

La construction du bief de la centrale hydraulique de Fessenheim (localité proche de Colmar) a nécessité le pompage de la nappe souterraine qui, une fois les travaux terminés, n'a pas repris son niveau initial.

Nous avons étudié sur ce site un peuplement de pins sylvestres, âgés de 7o ans, dont l'environnement a été ainsi modifié. Nous l'avons comparé à un peuplement de même espèce et de même âge, mais qui s'était développé en forêt de Charmes sous un cli nat et sur un sol différents de celui de la Hardt.

La construction du canal d'amenée de la centrale n'a pas eu, sur la structure des accroissements annuels des pins, un effet aussi marqué que prévu (à peine peut-on signaler une amélioration des coefficients de corrélation entre les données de Charmes et les données de Fessenheim lorsqu'on élimine les quatre années correspondant à la construction du bief!). Sans doute cet état de chose tient-il à la faible influence que peut exercer une variation de niveau d'une nappe qui oscille entre 4,80 et 9,20 $\mathrm{m}$ sur des arbres dont l'essentiel de l'enracinement s'arrête aux environs de $2 \mathrm{~m}$ !

Cependant, cette étude a permis de faire un certain nombre d'observations intéressantes en matière de dendrochonologie et de dendroclimatologie dont il est rendu compte ci-dessous.

\section{A. - DENDROCHRONOLOGIE,}

\section{I. - INTRODUCTION}

Dans cette première partie, nous allons comparer les deux peuplements de pins, nous attachant plus spécialement à les étudier du point de vue des années caractéristiques qu'ils présentent, et de leurs coefficients de sensibilité, d'autocorrélation et 
d'interdatation. Pour la sensibilité et l'autocorrélation, ces coefficients, comme nous le verrons, ne nous ont pas apporté toute la satisfaction que nous en attendions; leur manipulation a même semé le doute sur la façon traditionnelle de les utiliser.

\section{2. - MATÉRIEL E'T MÉTHODE \\ 2. 1. - Situation écologique}

Les arbres poussent sur deux stations bien différentes au point de vue phytosociologique et pédologique :

Station de Fiessenheim: (enrésinement d'un ancien taillis sous futaie).

\section{Végétation:}

- strate arborescente : Pinus silvestris, Quercus pedunculata:

- strate arbustive : Ligustrum vulgare*, Quercus pedunculata, Crateaegus monogyna*, Lonicera xylosteum*, Cornus sanguinea*, Pinus silvestris:

- strate herbacée: Avena elatior, Helianthemum vulgare.

Les arbres ont une faible hauteur : $13 \mathrm{~m}$ pour les pins; leur profondeur d'enracinement, déterminée par examen du profil des sols, est de l'ordre de r,80 m à 2 m (Bonveat et al., r963).

Sol (fig. I) :

$A_{1}(0-$ Io cm) gris-noir, argilo-limoneux, sans cailloux, granuleux.

$A_{1}^{\prime}($ - Io - $30 \mathrm{~cm})$ brun clair, limono-sableux, sans cailloux, parcouru par de grosses racines.

$\mathrm{C}_{1}(-30-90 \mathrm{~cm})$ graviers grossiers, sans racines.

$C_{2}(-90-\mathbf{I} 80 \mathrm{~cm})$ graviers avec 30 p. Ioo de limons sableux (Boxineau et al., 1963, op. cit.). 40 $\mathrm{cm}$.

Dans certains endroits, $A_{1}$ avait disparu, ou bien $A_{1}+\lambda_{1}$ variaient en épaisseur de ro à

Station de Charmes (premier boisement sur ancien terrain de culture).

\section{Végétation:}

- strate arbustive : Pinus silvestris;

- strate arborescente : Quercus pedunculata;

- strate herbacée (par ordre d'importance) : Rubus fruticosus, Polystichum spinulosum, Deschampsia coespitosa. Ces deux dernières espèces sont neutroacidiphiles et hygrophiles.

Les pins ont une hauteur de 20 à $25 \mathrm{~m}$.

Sol: c'est un pseudogley sur fragipan.

$A_{0}$ : moder légèrement hydromorphe, très noir, structure grumeleuse.

$A_{11}(0-3 \mathrm{~cm})$ brun gris, texture limoneuse.

$A_{12}(-3-5 \mathrm{~cm})$ beige, texture limoneuse, enracinement important et horizontal.

$A_{2}(-5-I 5 \mathrm{~cm})$ grisâtre, texture limoneuse, tassé, nombreuses concrétions rouilles et noires très dures ; racines horizontales, présence de galets siliceux, limite progressive avec 1'horizon sous-jacent.

$\mathrm{A}_{2} g(-\mathrm{I} 5-40 \mathrm{~cm})$ brun grisâtre, texture limono-argileuse, horizon fortement tassé, enracinement pratiquement nul; taches ocres augmentant avec la profondeur ; limite nette avec l'horizon inférieur.

* Espèces calcicoles. 


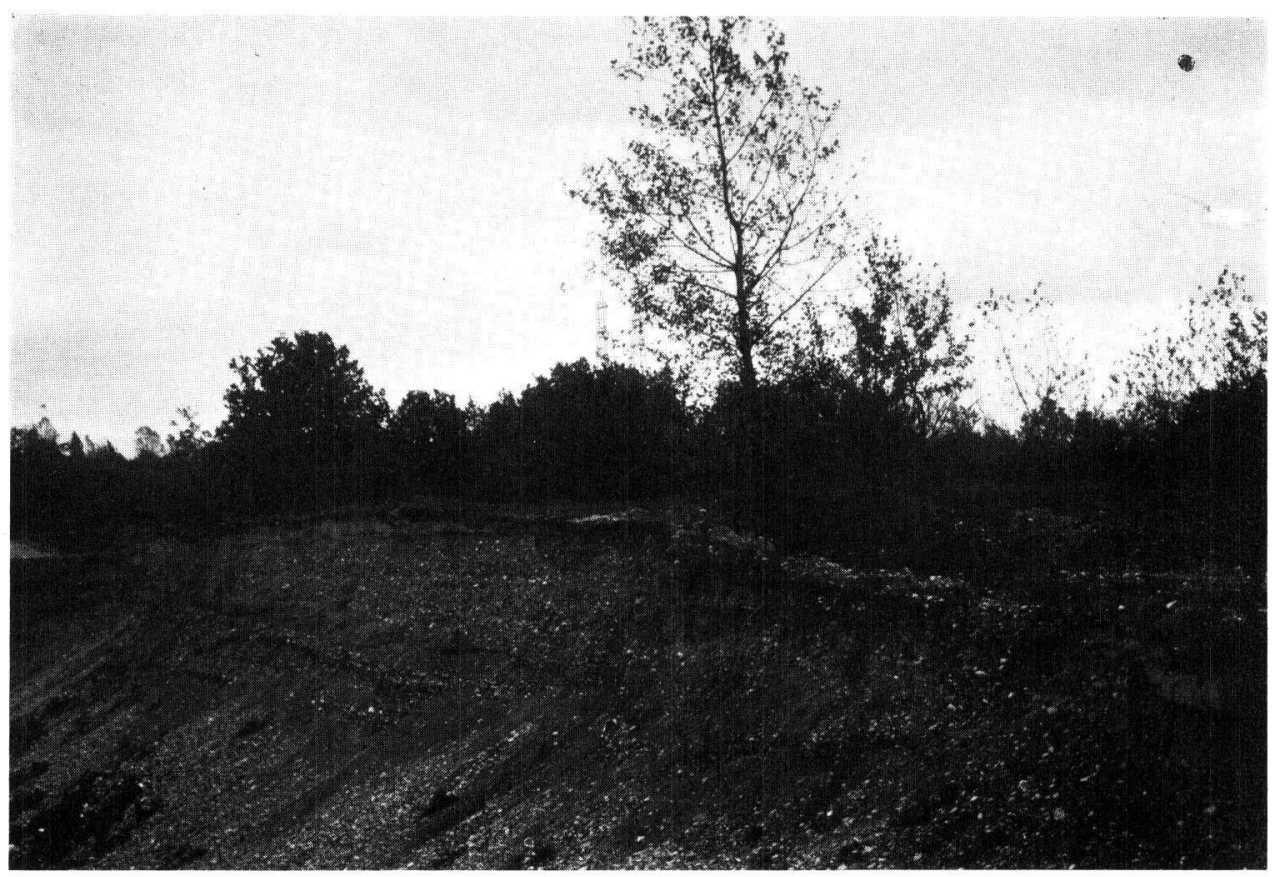

FIG. I. - Aspect du profil pédologique de la station de Fessenheim

The pedological profile of the Fessenheim Station

$\mathrm{B}_{g}\left(-\right.$ to $\left._{\mathrm{O}} \mathrm{IOO} \mathrm{cm}\right)$ : brun-ocre ; texture argileuse, structure polyédrique, quelques bandes décolorées verticales; absence de concrétions. (-IOo - I $30 \mathrm{~cm})$ : brun-ocre ; texture argilo-sableuse ; horizon de transition. inférieur à I fo cm : texture argilo-sableuse; nombreuses trainées verticales blanchâtres dans une matrice brune olivâtre; horizon de fragipan $(\mathrm{Bx})$. Limite inférieure du profil non atteinte à la tarière (I60 cm) (A. Brethes, I973).

Comme à Fessenheim, le sol renferme une nappe souterraine ; celle-ci est perchée et de plus temporaire (I. BECKER, I97I).

\section{2. - Échantillonnage et caractéristiques étudiées}

Dans chaque peuplement, nous avons prélevé sur 25 arbres dominants une carotte de $5 \mathrm{~mm}$ de diamètre ; les mesures des composantes de la densité du bois ont été faites par analyse densitométrique de radiographies des échantillons (H. PolgE, I966). De la lecture des courbes densitométriques ainsi obtenues, nous avons tiré 9 variables par arbre et par année, ceci pendant 50 ans; ce sont (fig. 2) :

- la densité maximale D exprimée en $\mathrm{g} / \mathrm{dm}^{3}$;

- la densité minimale $d$ en $\mathrm{g} / \mathrm{dm}^{3}$;

- la largeur totale du cerne $\mathrm{L}$ en $\mathrm{mm}$;

- la largeur de bois dont la densité est égale à $550 \mathrm{~g} / \mathrm{dm}^{3}$, soit $l_{550} \mathrm{en} \mathrm{mm}$;

- la largeur de bois à mi-hauteur entre $\mathrm{D}$ et $d$, soit $l \mathrm{~h} / 2 \mathrm{en} \mathrm{mm}$;

- le pourcentage de bois dont la densité est supérieure ou égale à foo $\mathrm{g} / \mathrm{dm}^{3}$, soit $\mathrm{P}_{\mathbf{4 0 0}}$, $550 \mathrm{~g} / \mathrm{dm}^{3}$, soit $\mathrm{P}_{550}$, $700 \mathrm{~g} / \mathrm{dm}^{3}$, soit $\mathrm{P}_{700}$;

- la densité moyenne en $\mathrm{g} / \mathrm{dm}^{3}$ : D moy. 


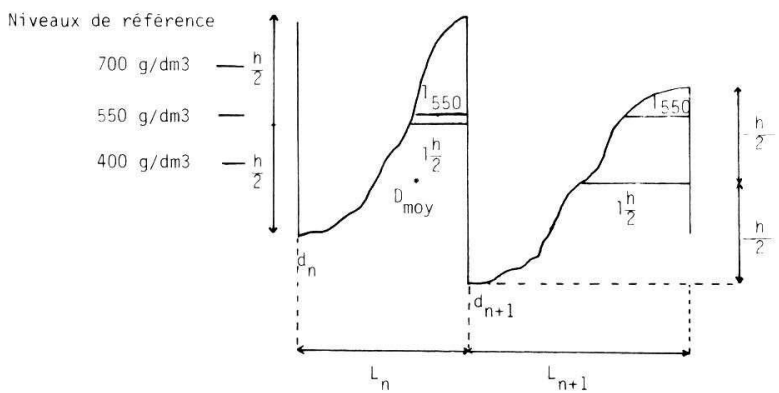

Fig. 2. - Localisation de quelques variables sur les cernes des années $n$ et $n+1$

The localization of some variables upon age-rings of the years $\mathrm{n}$ and $\mathrm{n} \quad \mathrm{I}$

\section{La densité moyenne (fig. 3).}

La densité movenne d'un cerne est obtenue en divisant la surface comprise entre la courbe et une ordonnée de référence par la largeur d'accroissement; faute de connaître de façon exacte cette surface, nous en avons calculé une valeur approchée en assimilant le profil de densité à une série de 4 segments de droite reliant le point de la densité maximale au point de la densité minimale, en passant par les points d'intersection de la courbe avec les trois niveaux de référence de densité.

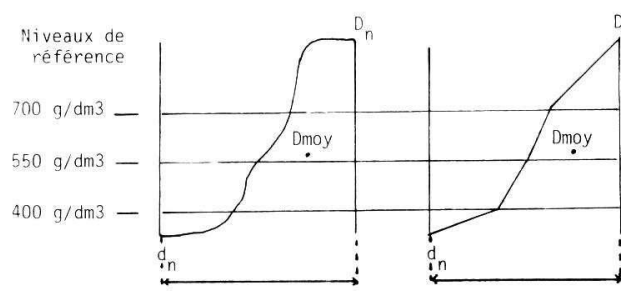

Fig. 3. - La densité moyenne

Average density

\section{3. - RÉsultates}

Afin de situer l'ordre de grandeur des variables dendrochronologiques, nous avons établi un tableau récapitulatif donnant leurs valeurs moyennes et l'écarttype pour chaque peuplement.

\section{I. - Les années caractéristiques}

Les années caractéristiques sont les années où 20 arbres sur 25 au moins présentent pour une variable une valeur, soit plus élevée, soit moins élevée que l'année précédente, cette valeur différant dans un sens ou dans un autre d'au moins :

- $0,52 \mathrm{~g} / \mathrm{dm}^{3}$ pour la densité minimale et maximale,

- I/IOoe de $\mathrm{mm}$ pour la largeur de cerne,

- I p. Ioo pour les différents pourcentages. 
TABLEAU I

Tableau des valeurs movennes

et écart-type des données du bois de chaque peuplement

Table of the average values and standard deviation of wood variables in each population

\begin{tabular}{|c|c|c|c|c|c|c|c|c|c|c|}
\hline \multirow{2}{*}{ Stations } & & \multicolumn{9}{|c|}{ Variables } \\
\hline & & 1) & $d$ & $\mathrm{~L}(\mathrm{~mm})$ & $P_{400}$ & $P_{550}$ & $P_{6 C 0}$ & $\begin{array}{c}l_{550} \\
(\mathrm{mmm})\end{array}$ & $l_{2}^{h}(\mathrm{~mm})$ & Dmos \\
\hline \multirow{2}{*}{ Fessenheim } & Moyenne .... & 771 & 259 & 1,51 & $4: 3$ & $2 x$ & 17 & 0,12 & 0,49 & 456 \\
\hline & Ecart-type .. & 67 & 18 & 0,35 & 7 & 8 & 8 & 0,19 & 0,15 & 36 \\
\hline \multirow{2}{*}{ Charmes } & Moyenne .... & 792 & 253 & 1,93 & 49 & 34 & 19 & 0,67 & 0,76 & $47 i$ \\
\hline & Ecart-type .. & 40 & 18 & 0,40 & 57 & 6.2 & 50 & 0,18 & 0,23 & 23 \\
\hline
\end{tabular}

Sur chaque profil synthétique (fig. 4), nous les avons fait apparaître par un trait, liant l'année caractéristique à la précédente pour trois variables $\mathrm{D}$, $d$ et $\mathrm{L}$; en réalité, nous les avons déterminées pour les neuf variables (fig. 5 et 6).
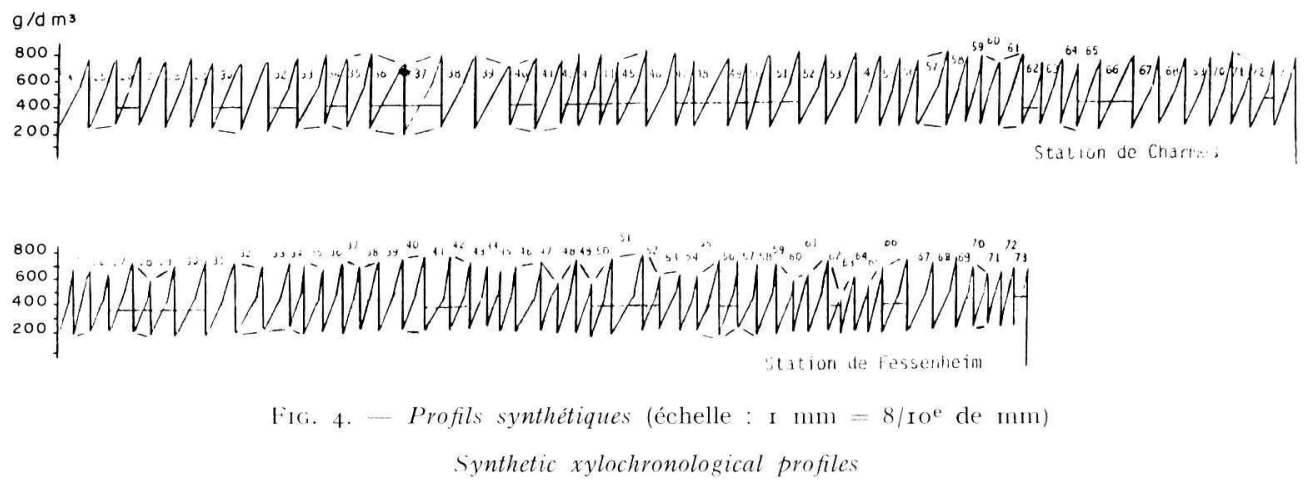

W'après les figures 5 et 6 , nous pouvons faire les observations suivantes :

- pour une même variable, le nombre des années caractéristiques varie d'une station à l'autre,

- si une année s'avère caractéristique pour les deux stations, il arrive parfois que le sens de variation change de l'une à l'autre (exemple : en I 935 la densité maximale),

- la densité maximale est plus discriminante que la largeur du cerne à Fessenheim, mais à Charmes, nous avons l'inverse,

- sur 50 années étudiées, $n$ années sont caractéristiques pour au moins une variable dendrochronologique $(n=39$ à Fessenheim contre 44 à Charmes) ; pour 


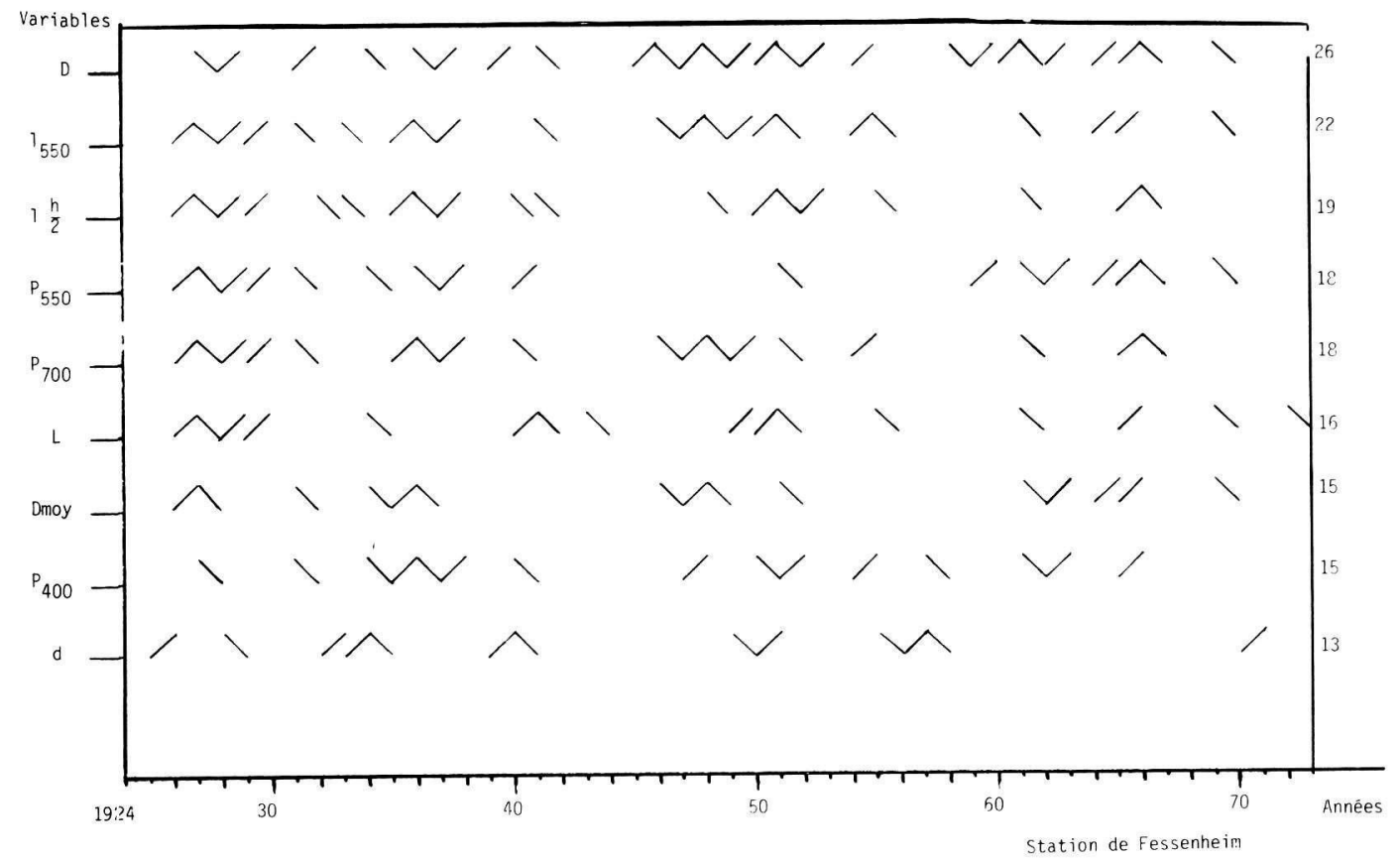

FIG. 5. - Récapitulation des années caractéristiques et de leur évolution données par les différentes variables A comprehensive table of characteristic years and of their evolution given by different iariables

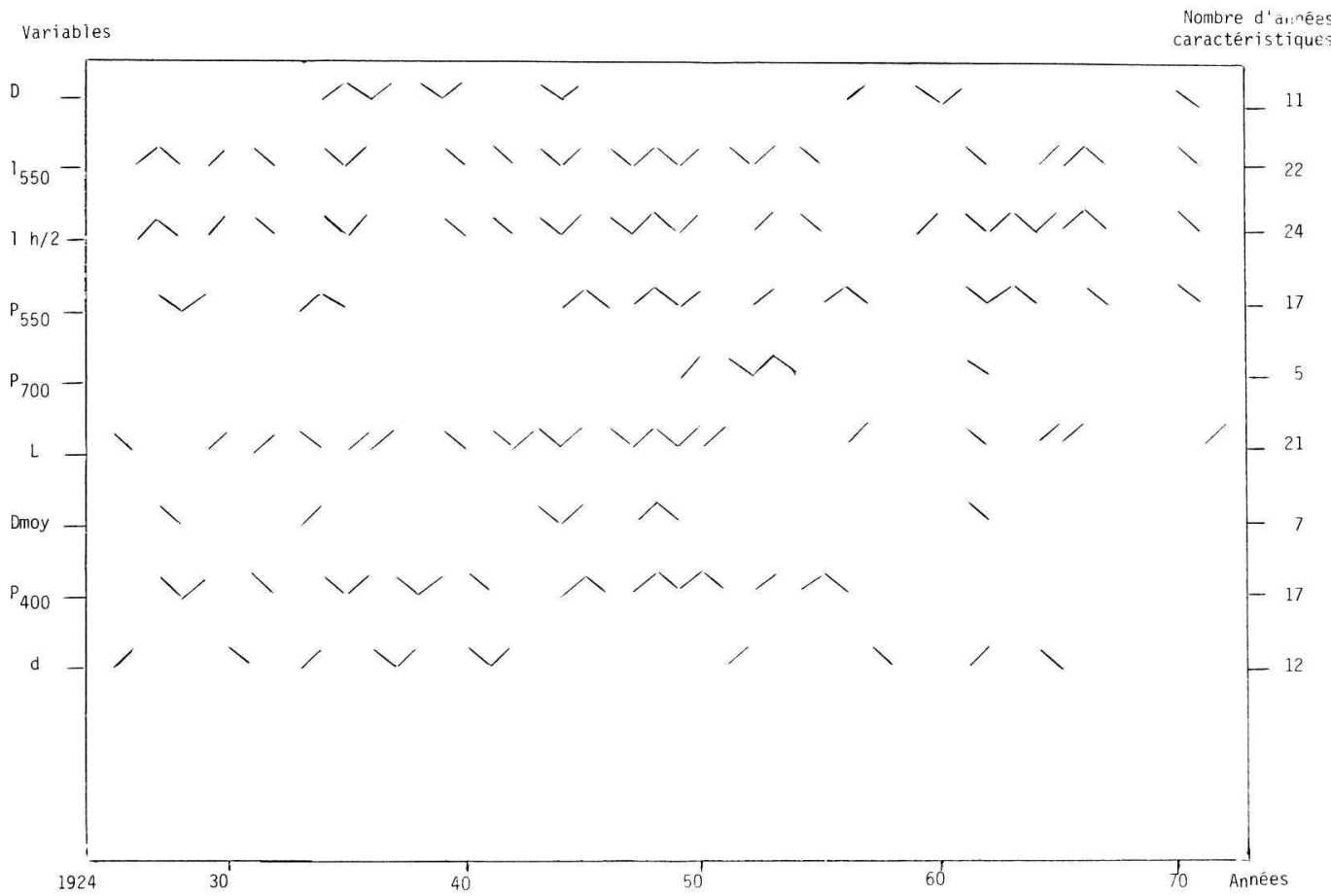

FIG. 6. - Récapitulation des années caractéristiques et de leur évolution données par les différentes variables A comprehensive table of characteristic vears and of their evolution given by different iariables 
chaque peuplement, nous avons évalué le rapport entre le nombre des années caractéristiques pour une variable précise et $n$ correspondant à la même station ; la grande différence entre les deux stations tient à la densité maximale et au pourcentage $P_{700}$ pour lesquels à Fessenheim nous avons respectivement 2,68 et 4,I 8 plus d'années caractéristiques qu'à Charmes (tabl. 2).

\section{TABI, EAU 2}

Rapport entre le nombre d'années caractéristiques donné par une variable sur le nombre total d'années caractéristiques de la même station

The relation between the number of characteristic years, given by a variable, and the total number of the characteristic years in the same station

\begin{tabular}{|c|c|c|c|}
\hline \multirow[t]{2}{*}{ V'ariables } & \multicolumn{2}{|c|}{$\begin{array}{l}\text { Nombre d'années caractéristiques } \\
\text { donné par une variable } n\end{array}$} & \multirow{2}{*}{$\frac{A_{1}}{A_{2}}$} \\
\hline & Fessenheim $\left(A_{1}\right)$ & Charmes $\left(A_{2}\right)$ & \\
\hline I) & $(0,67$ & 0,25 & 2,48 \\
\hline$d$ & 0,33 & 11,27 & 1,22 \\
\hline $\mathrm{L}$. & $0, t_{1}^{\prime}$ & 0,48 & $11, \times \prime^{\prime}$ \\
\hline 1) $)_{\operatorname{moy}}$ & 11,38 & 11,16 & 2,38 \\
\hline$l_{2}^{h}$ & 0,49 & 0,55 & 0,89 \\
\hline$l_{550}$ & 0,56 & 0,50 & 1,12 \\
\hline$P_{400}$ & 0,38 & 0,39 & 0,97 \\
\hline $\mathrm{P}_{550}$ & 0,16 & $1), 39$ & 1,17 \\
\hline$P_{700}$ & 0,16 & 0,11 & 4,18 \\
\hline
\end{tabular}

\section{2. - La sensibilité et l'autocorrélation}

Cette étude nous offrait la possibilité de caractériser chaque peuplement de façon qualitative du point de vue dendrochronologique. Nous avons calculé les coefficients de sensibilité C et d'autocorrélation ?

$$
\begin{aligned}
& \mathrm{C}=\frac{\mathrm{I}}{n-\mathrm{I}} \sum_{i=1}^{n-1} 2 \frac{\left|x_{i+1}-x_{i}\right|}{\left|x_{i+1}+x_{i}\right|} \\
& \mathrm{P}=\sum_{i=1}^{n-1}\left(x_{i+1}-x\right)\left(x_{i}-x\right) /\left(x_{i}-x\right)^{2}
\end{aligned}
$$

$x=$ valeur de la variable étudiée :

$i=$ indice de l'année.

Le coefficient de sensibilité traduit l'évolution de l'année $n+$ I par rapport à l'année $n$. Plus sa valeur sera élevée, plus le petıplement offrira une bonne base pour une étude dendrochronologique. Pour le coefficient d'autocorrélation, qui donne le degré de corrélation entre les années $n$ - I et $n$, c'est l'inverse : une valeur élevée de p est vue de façon défavorable par les dendrochronologistes, car si les années successives sont fortement liées entre elles, elles ne pourront pas être très discriminantes pour la datation du hois. 
D'après les auteurs américains M. L. PARker et W. E. S. Hexoch (I97I), deux méthodes de calcul ont été envisagées pour $\mathrm{C}$ et $\rho$ à partir :

- des valeurs mesurées ;

- des indices.

Les valeurs mesurées sont celles lues sur les courbes densitométriques qui constituent les données du profil synthétique. Les indices ont été utilisés dans le cas où l'on a cherché à éliminer une tendance générale en fonction du temps.

Lorsque le coefficient de corrélation $r$ entre le numéro de l'année et la variable mesurée était significatif, nous avons déterminé l'équation de régression linéaire de cette variable en fonction du temps, soit:

$$
\mathrm{Y}=a \mathrm{X}+b
$$

où : $\mathrm{X}=$ numéro de l'année

$\mathrm{Y}=$ variable étudiée

$a=$ pente de la droite qui chiffe la tendance

$b=$ constante.

$I^{\circ \mathrm{r}}$ cas : $r$ n'est pas significatif :

$$
\text { indice }=\frac{x}{x}
$$

$x=$ valeur mesurée de la variable.

$x=\frac{x}{50}$

2 e cas: $r$ est significatif

$$
\text { indice }=\frac{x}{x_{r}}
$$

$x=$ valeur mesurée de la variable,

$x_{c}=$ valeur calculée de cette même variable à partir de la droite de régression $\mathrm{X}_{c}=a \mathrm{X}+b, \mathrm{X}$ étant le numéro de l'année, ici $\mathrm{I} \leqslant \mathrm{X} \leqslant 5^{\circ}$.

Les valeurs obtenues pour ces coefficients varient suivant que le calcul est effectué à partir de valeurs brutes ou indicées (exemple : la largeur de cerne à Fessenheim), mais ce phénomène n'est pas général (exemple : la densité minimale à Fessenheim où la suppression de la tendance n'a pratiquement pas eu d'effet sur la valeur de C).

Les conclusions que l'on pourrait être amené à donner en examinant C et $P$ sont en contradiction avec celles fournies par l'emploi des années caractéristiques qui représente une méthode simple à mettre en ceuvre et d'interprétation facile.

Exemple: A Charmes, si nous ne tenons compte que des trois densités, la densité moyenne apparait comme la variable la plus sensible ; lui font suite la densité minimale et la densité maximale (tabl. 3) ; or si nous regardons la figure 6 , nous obtenons un ordre différent : la densité minimale offre le plus d'années caractéristiques, puis vient la densité maximale et enfin la densité moyenne.

Autre observation étonnante tirée du peuplement de Charmes : $P_{550}$ est une variable montrant une sensibilité élevée, mais aussi une autocorrélation importante.

Même en éliminant la tendance, le résultat de ces calculs demanderait done à être réexaminé. Il semble que $\mathrm{C}$ et p puissent servir à comparer utilement une même 
variable entre deux peuplements ou plus; mais les coefficients ne devraient être utilisés qu'avec beaucoup de prudence pour comparer diverses variables dans un même peuplement. Ceci est peut-être dû à la variabilité des divers critères étudiés : la densité maximale par exemple n'a jamais doublé de valeur au cours des 50 années étudiées, comme c'est le cas pour la largenr de cerne, et il est donc hasardeux de comparer des coefficients à partir de caractéristiques qui évoluent de façon si différente.

\section{I'ABLEAU 3}

Tableau des valeurs de C et de?

Recapitulation table of the $\mathrm{C}$ and $\mathrm{p}$ values

Variables

$d^{* *}$
$\mathrm{D}^{* *}$
$\mathrm{~L}^{* *}$
$\mathrm{D}_{\operatorname{moy}}{ }^{* *}$
$\mathrm{P}_{400}(\chi d \geqslant 400)$
$\mathrm{P}_{550}(\chi d \geqslant 55(1)$
$\mathrm{P}_{700}(\chi d \geqslant 700)$

$d *$

L**

$1)_{\operatorname{moy}}$

$P_{400}(\chi d \geqslant 400)$

$\mathrm{P}_{550}(\chi d \geqslant 550)$

$\mathrm{P}_{7,10}(y d \geqslant 700)$ c

C indice

$p$

$p$ indice

Tendance en fonction du vieillissement de l'arbre :

* tendance positive

** tendance négative.

Seuils de signification de p (table des corrélations de Fisher) :

$$
\begin{array}{rl}
* & 0,1 \\
* * & 0,05 \\
* * * & 0,01 \\
* * * * & 0,001 .
\end{array}
$$

\begin{tabular}{|c|c|c|c|c|}
\hline 0,0590 & & 0,0592 & $0,5081 * * * *$ & 0,1943 \\
\hline 0,0$) 131$ & & $0,(1)^{\prime}+34$ & $-1,0295$ & $-0,0298$ \\
\hline 0,2015 & & $11,-2039$ & $0,3791 * * *$ & 0,$2966 ; * *$ \\
\hline 0,$06 ; 2$ & & $0,06.522$ & $-1,31: 37 * *$ & $0,3248 * *$ \\
\hline $0,158:$ & 1 & 0,1582 & $-0,3920 * * *$ & $0,3898 * * *$ \\
\hline $0,2 \div 19$ & & 0,2919 & $-0,31553^{* * *}$ & $0,3437 * * *$ \\
\hline $0,33 \times 9$ & & 0,3389 & $-1,26339 *$ & $-11,26336 *$ \\
\hline
\end{tabular}

Station de Fessenheim

$\begin{array}{llll}0,0608 & 0,0610 & 0,2496 * & 0,7993^{* * * *} \\ 0,0887 & 0,0875 & 0,0977 & 0,0200 \\ 0,1705 & 0,3134 & 0,5272 * * * * & 0,4067 * * * * \\ 0,091: & 11,0916 & 0,0192 & 0,0305 \\ 0,1999 & 11,1901 & 0,0965 & -0,0959 \\ 0,376,4 & 0,3764 & 0,0830 & -0,0830 \\ 0,6184 & 11,6197 & 0,0484 & -0,0586\end{array}$

Station de Charmes

En matière de méthodologie, ceci amène à mettre en cause l'intérêt des coefficients C et $\rho$ pour l'étude de caractéristiques autres que les largeurs de cernes: lorsque l'on veut tester le pouvoir discriminant de diverses variables, l'utilisation des années caractéristiques semble au vu de cette étude beaucoup plus sûre. 


\section{4. - L'INTERDATATION}

Le coefficient d'interdatation $\mathrm{R}$ donne le degré d'homogénéité d'un peuplement clu point de vue de la datation des arbres qui le composent :

$$
\mathrm{R}=\frac{\mathrm{C}_{m}}{\frac{\mathrm{C}_{i}}{n}}
$$

R est le rapport entre la sensibilité de l'arbre moyen $C_{m}$ et la moyenne de sensibilité des individus qui la composent $\frac{\mathrm{C}_{i}}{n}$.

Si les arbres formant le peuplement présentent des variations synchrones parfaites $\mathrm{R}=\mathrm{I}$; dans le cas contraire $: \mathrm{R}=\frac{\mathrm{I}}{\sqrt{n}}, n=25$, soit $\mathrm{R}=0,2(\mathrm{~A}$. I. Munaut, I $(0) 60)$.

Les valeurs $\mathcal{C}_{m}$ et $\mathcal{C}_{\text {; }}$ sont celles calculées sur les valeurs brutes; nous avons obtenu :

$$
\text { TABI.EAL }+
$$

Laleurs du coefficient d'interdatation

\begin{tabular}{|c|c|c|}
\hline Variables & I'cssenheim & Charmes \\
\hline I) & 0,736 & $(1,5336$ \\
\hline$d$ & 0,577 & 0.633. \\
\hline $\mathrm{L}$ & 0,633 & 0,$766 ;$ \\
\hline $\mathrm{D}_{\text {mox }}$ & $0,7 \times 1$ & 0,675 \\
\hline$P_{100}$ & 0,6661 & 0,650 \\
\hline$P_{550}$ & $0,6 x=$ & $0,6.5 .3$ \\
\hline$P_{700}$ & $0,69.3$ & $(0,5 ., 2)$ \\
\hline
\end{tabular}

Cross-dating values

Par le nombre des années caractéristiques qu'elles nous donnaient, nous avions classé les variables, considérant comme la plus discriminante celle où leur nombre était le plus important. Nous pouvons considérer que cet ordre est peu différent de celui obtenu ici avec le coefficient d'interdatation.

Dans l'ensemble, les pins ont fourni des résultats très comparables d'une station à l'autre. Cependant, à Charmes, la largeur du cerne offre un coefficient $\mathrm{R}$ plus élevé qu'à Fessenheim $(0,766$ contre 0,638$)$. Peut-être peut-on voir là l'effet de la sylviculture! Dans le peuplement vosgien, les arbres semblent aroir fait l'objet d'un soin 
particulier, ce qui a pu conduire à une certaine homogénéité dans leur croissance diamétrale. A Fessenheim, au contraire, on rencontre une proportion notable d'arbres mal formés et qui ont poussé assez peu. Pour les autres variables, on serait tenté de conclure que Fesenheim offre une plus grande homogénéité. Les pins en Alsace sont soumis à des conditions plus rudes et aussi plus tranchées et de ce fait, aucun arbre ne peut rester insensible à de telles variations. A Charmes, sous un climat plus tempéré, la réaction des arbres est plus individuelle.

\section{5. - Conclusion}

Plusieurs méthodes ont été envisagées pour l'étude de ces deux peuplements : nous n'en retiendrons que deux (les coefficients d'autocorrélation et de sensibilité offrant des résultats incertains) :

- les années caractéristiques,

- l'interdatation.

Les années caractéristiques traduisent la sensibilité de l'ensemble des arbres de chaque station, quelle que soit l'amplitude de la réponse de l'arbre à un facteur : nous avons ici la traduction de la sensibilité qualitative.

L'interdatation nous montre la simultanéité des réactions des divers individus d'un même peuplement en fonction d'événements extérieurs. Nous arons ainsi la sensibilité d'un point de vue quantitatif.

Associant les résultats obtenus par les années caractéristiques et l'interdatation, nous pouvons conclure que le peuplement de Fessenheim est plus sensible que celui de Charmes (i62 années caractéristiques contre I36 à Charmes).

Cette remarque peut trouver une explication dans l'influence du climat qui va faire l'objet d'une étude dans la seconde partie, mais peut-être aussi par le sol et la sylviculture.

A Fessenheim, nous avons, sous un climat relativement sec, un sol filtrant; les arbres manquant d'eau captent celle-ci lors de son passage, donc pendant un temps très limité qui est sensiblement le même pour tous les arbres. A Charmes, au contraire, le pseudogley constitue une réserve d'eau qui sera utilisée par l'arbre en fonction de ses besoins propres ou de ses capacités d'absorption.

Ceci pourrait expliquer qu'à Charmes, la croissance ait été plus importante qu'à Fessenheim, en raison à la fois de la capacité de rétention du sol et des éclaircies qui ont été pratiquées; les effets de concurrence se trouvent ainsi réduits au niveau du houppier (1umière) et au niveau du sol (eau, substances minérales), et chaque arbre a pu se développer suivant son dynamisme propre.

\section{B. - DENDROCLIMATOLOGIE}

\section{I. - INTRODUCTION}

La chaîne des Vosges, perpendiculaire aux vents d'Ouest, crée dans la région de la Hardt, le climat le plus continental de France ; à Charmes, nous avons un climat semi-continental. 
Pour ces deux stations, nous avons voulu connaître l'influence du climat sur la croissance des pins, en y ajoutant, dans le cas de Fessenheim, l'effet de l'abaissement de la nappe.

\section{2. - MATÉRIEL ET MÉTHODF:}

2. I. Données climatologiques de base

Station de Fessenheim.

Ces données nous ont été fournies par l'Aménagement hydraulique de la Plaine du HautRhin. Le tableau 5 donne une reproduction très partielle des indications dont nous avons disposé pour 50 ans (I924-I973):

- la température mensuelle moyenne exprimée en oC : TM,

- la pluviométrie mensuelle en $\mathrm{mm}$ : P, (en $\mathrm{mm}$ )

- l'évapotranspiration potentielle calculée à partir de la formule de Thorntwhaite : ETP

- les déficits globaux (exprimés négativement) ou les excédents globaux mensuels (exprimés positivement); ils représentent la différence : pluie - ETP.

De plus, pour 3 types de sols, différents l'un de l'autre par leur réserve utile (RU), qui sont respectivement: 35,70 et $140 \mathrm{~mm}$, nous avions :

- la valeur de la réserve en eau des trois types de sols à la fin de chaque mois; cette réserve est constituée par les excédents pluviométriques ( $\mathrm{P}-\mathrm{ETP}>\mathrm{o}$ ) cumulés jusqu'à la valeur de la réserve utile (RU) ; au-dessus de cette valeur, on considère qu'il y a drainage. Cette réserve est diminuée par les déficits globaux $(\mathrm{P}-\mathrm{E} T \mathrm{~T} P<\mathrm{o})$; à l'épuisement de la réserve, le sol est au point de flétrissement.

- les besoins en eau de complément (BO en $\mathrm{mm}$ ) ; ces besoins ont été considérés comme nuls tant que la réserve utile (RU) n'a pas été épuisée, c'est-à-dire tant que le déficit n'atteignait pas le point de flétrissement. Ils ont été obtenus en faisant la différence : déficit global du mois considéré $(\mathrm{P}-\mathrm{E} T \mathrm{P}<\mathrm{O})_{n}$ - réserve du mois précédent $(\mathrm{RU})_{n-1}$.

TABLEAU 5

Reproduction particlle du tableau des données climatiques

A partial reproduction of the table of climatic variables

\begin{tabular}{|c|c|c|c|}
\hline \multicolumn{4}{|c|}{ Année 19'9 } \\
\hline & Mars & Avril & Mai \\
\hline $\mathrm{TM}\left({ }^{\circ} \mathrm{C}\right) \ldots \ldots \ldots$ & 5,3 & $1: 3,9$ & 14,1 \\
\hline$P(m m) \ldots \ldots \ldots$ & 18,4 & 30,9 & 666,4 \\
\hline $\operatorname{ETP}(\mathrm{mm}) \ldots \ldots$ & 17 & 67 & 77 \\
\hline $\mathrm{P}-\mathrm{ETP}(\mathrm{mm}) \ldots$ & 1,4 & $-36,1$ & $-10,1$ \\
\hline $\mathrm{RU}_{35} \ldots \ldots \ldots \ldots$ & 35 & 0 & 0 \\
\hline $\mathrm{RU}_{70} \ldots \ldots \ldots$ & 55,1 & 19 & 8,1 \\
\hline $\mathrm{RU}_{140} \ldots \ldots \ldots \ldots$ & 55,1 & 19 & 8, \\
\hline $\mathrm{BO}_{35} \ldots \ldots \ldots \ldots$ & 11 & 1,1 & 11,1 \\
\hline $13 \mathrm{O}_{70} \ldots \ldots \ldots$ & (1) & 11 & 0 \\
\hline $\mathrm{BO}_{140} \ldots \ldots \ldots$ & $(1$ & 0 & 0 \\
\hline
\end{tabular}

Pour la nappe, nous avons obtenu des relevés piézométriques mensucls de 1952 à 1973 : cette nappe qui se trouve à une profondeur moyenne de $6 \mathrm{~m}$ a été maintenue à $8,70 \mathrm{~m}$ en moyenne pendant les travaux de 1953 à 1956 (fig. 7). 


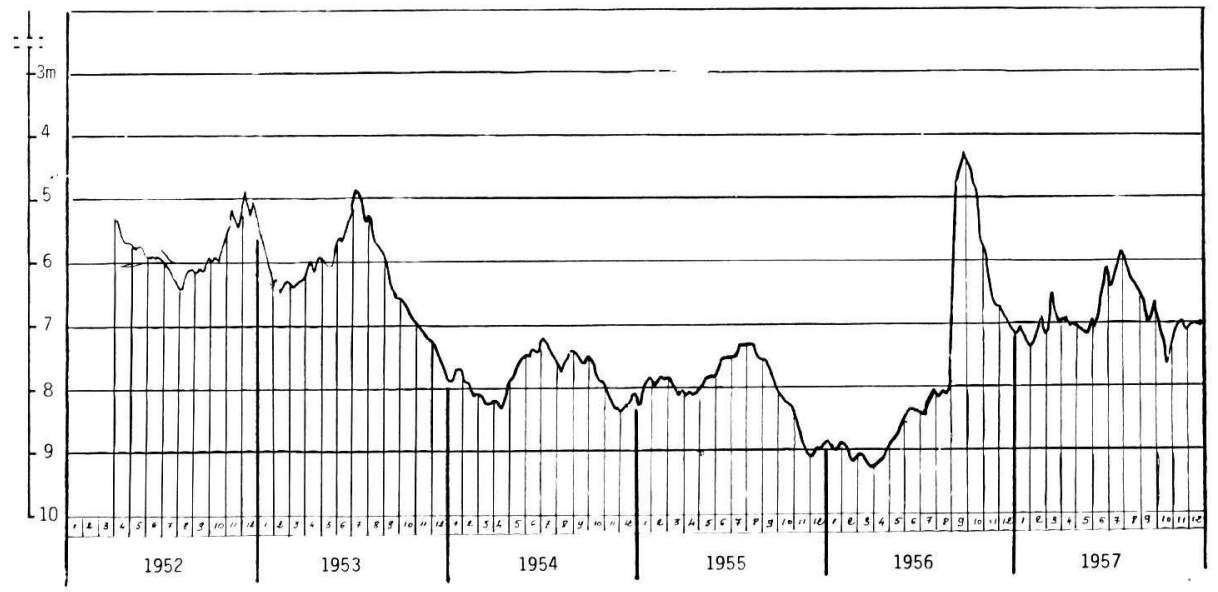

I:IG. 7. - Fluctuation du niveau de la nappe pendant les travaux; octobre 1953-septembre 1956 The fluctuation of the level of the water table during the work period; October 1953-September 1956i

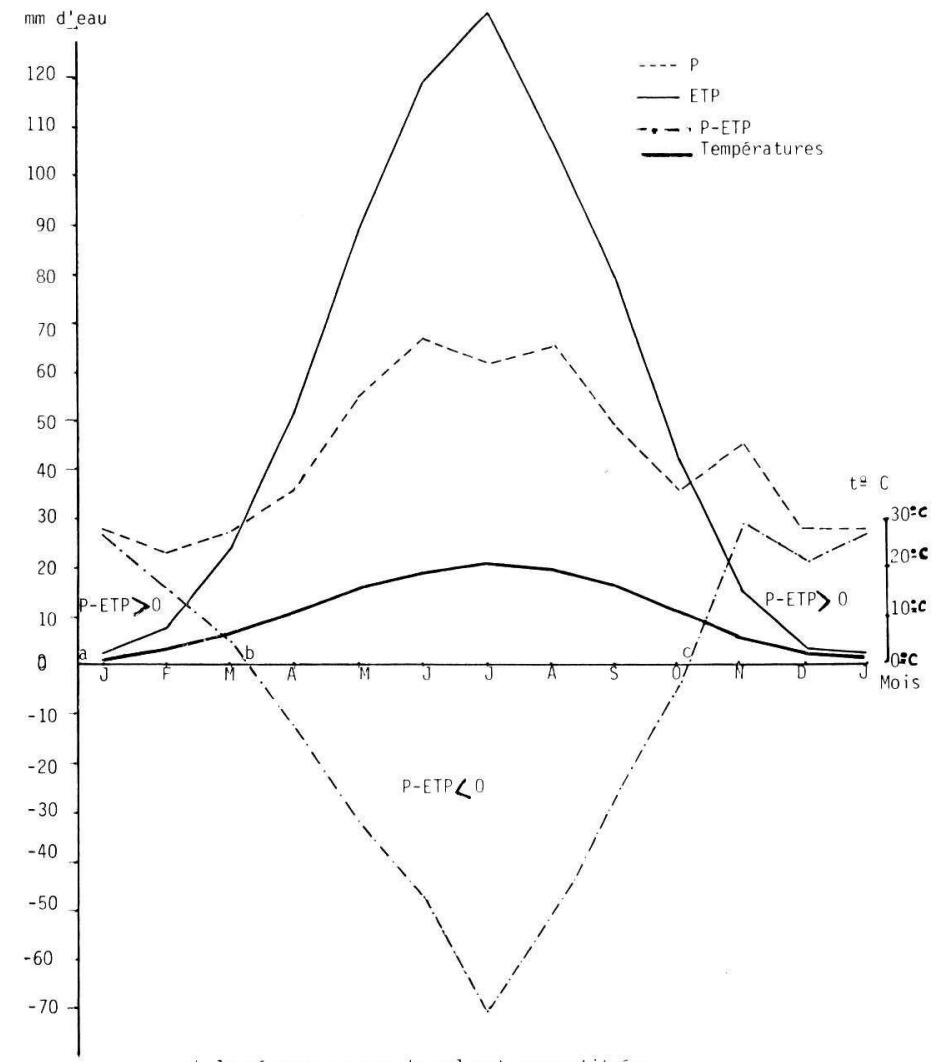

- ab la réserve en eau du sol est reconstituée

- bc épuisement des réserves du sol

- c reconstitution des réserves du sol

Bilan pour une période de 50 ans

FIG. 8. - Diagramme pluviothermique de la région de la Hardt

The balance sheet of the rainfall for Fessenheim and evolution of the average $C$ temperature over 50 years 


\section{Station de Charmes.}

Pour cette station, les relevés météorologiques étaient pratiquement inexistants. Nous avons

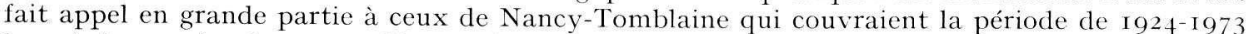
hormis les années de guerre. Nous avions ainsi :

- la pluviosité mensuelle: P (en mm),

- la température maximale mensuelle moyenne en oC : TA,

- la température minimale mensuelle moyenne en oC : TI.

Ces données nous ont permis d'établir pour chaque station un diagramme pluviothermique représentant le climat d'une année théorique (année moyenne des 5o étudiées) : figures 8 et 9 .

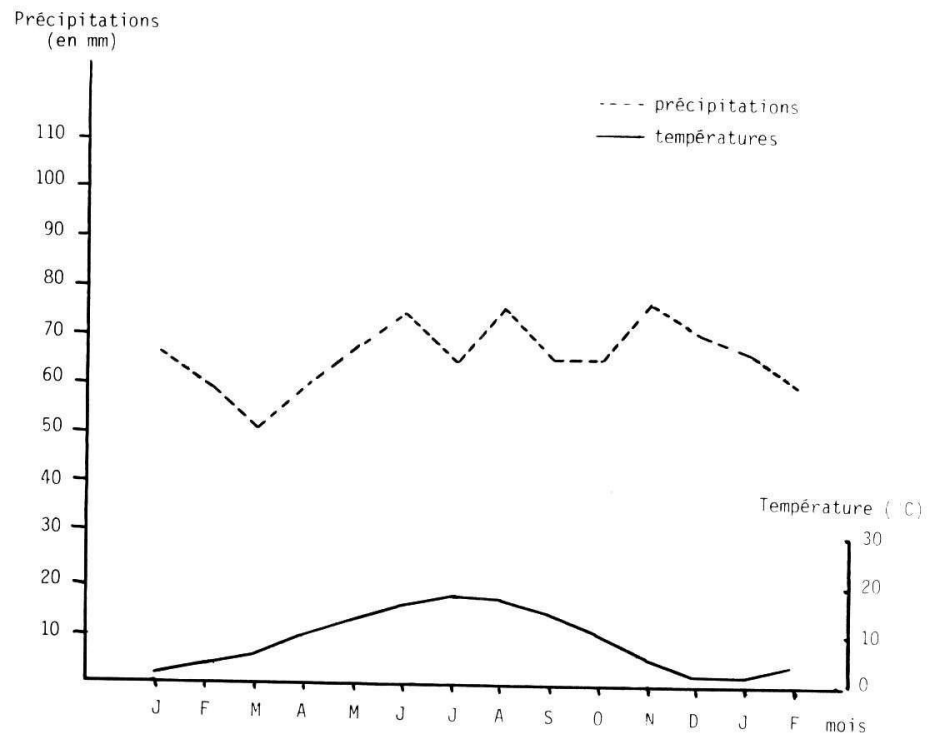

Fír. 9. - Représentation graphique des données climatiques pour une année théorique (région de Charmes)

A graphic representation of the climatic variables for one theoretic year

(Charmes)

$$
\text { 2. 2. - Méthode d'étude }
$$

Nous avons calculé le coefficient de corrélation existant entre une donnée dendrochronologique de l'arbre moyen et la somme des données climatologiques moyennes couvrant une période pouvant aller de I à 12 mois (tabl. 6). Pour la nappe, seule une période de un mois a été envisagée au départ.

\section{3. - RÉsultats}

\section{I. - Influence de la température}

A partir du tableau 7, différentes remarques s'imposent:

A Fessenheim, le seuil de signification du coefficient de corrélation n'atteint jamais I p. I ooo ; en revanche, à Charmes, il est atteint pour la densité minimale et $\mathrm{P}_{550}$. 
TABLEAU 6

Représentation schématique des 86 périodes de temps

servant au calcul des corrélations climat; données dendrochronologiques

Diagrammatic sketch of the 86 annual periods used for the correlation analysis

Mois de l'année $n-1$

Mois de l'année n

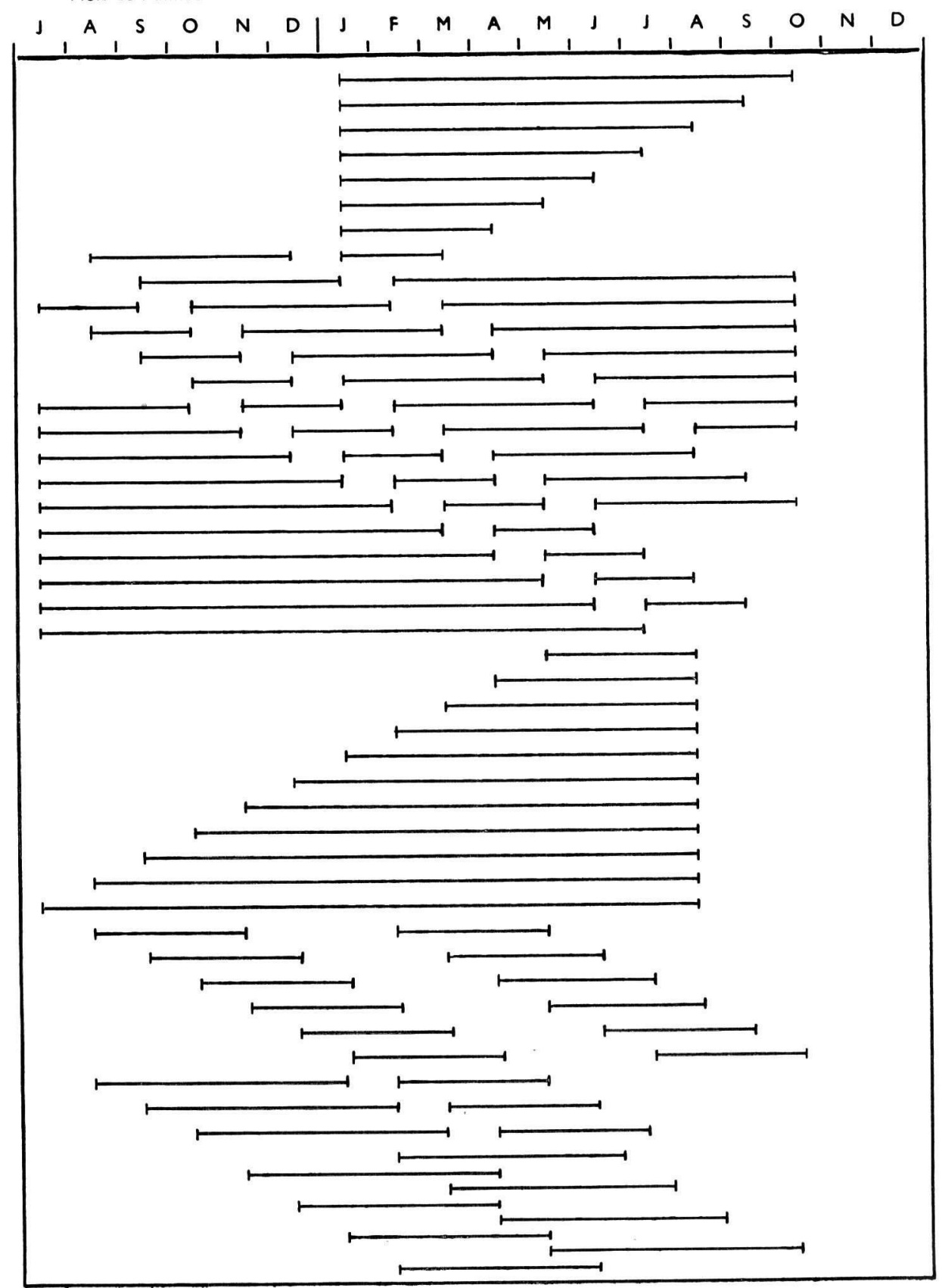


Bien que dans 1'ensemble, à Charmes nous atteignons des coefficients plus élevés qu'à Fessenheim, il est hasardeux de conclure car l'étude a été menée de façon différente suivant la station (à Fessenheim, les corrélations ont été faites à partir des données mensuelles moyennes; à Charmes, à partir de température minimale et maximale moyenne mensuelle).

\section{TABLEAU 7}

Étude de la corrélation entre la température d'une période définie et les différentes variables de l'année $n$

The correlation between the temperature

of a given period and the different variables in the year $n$

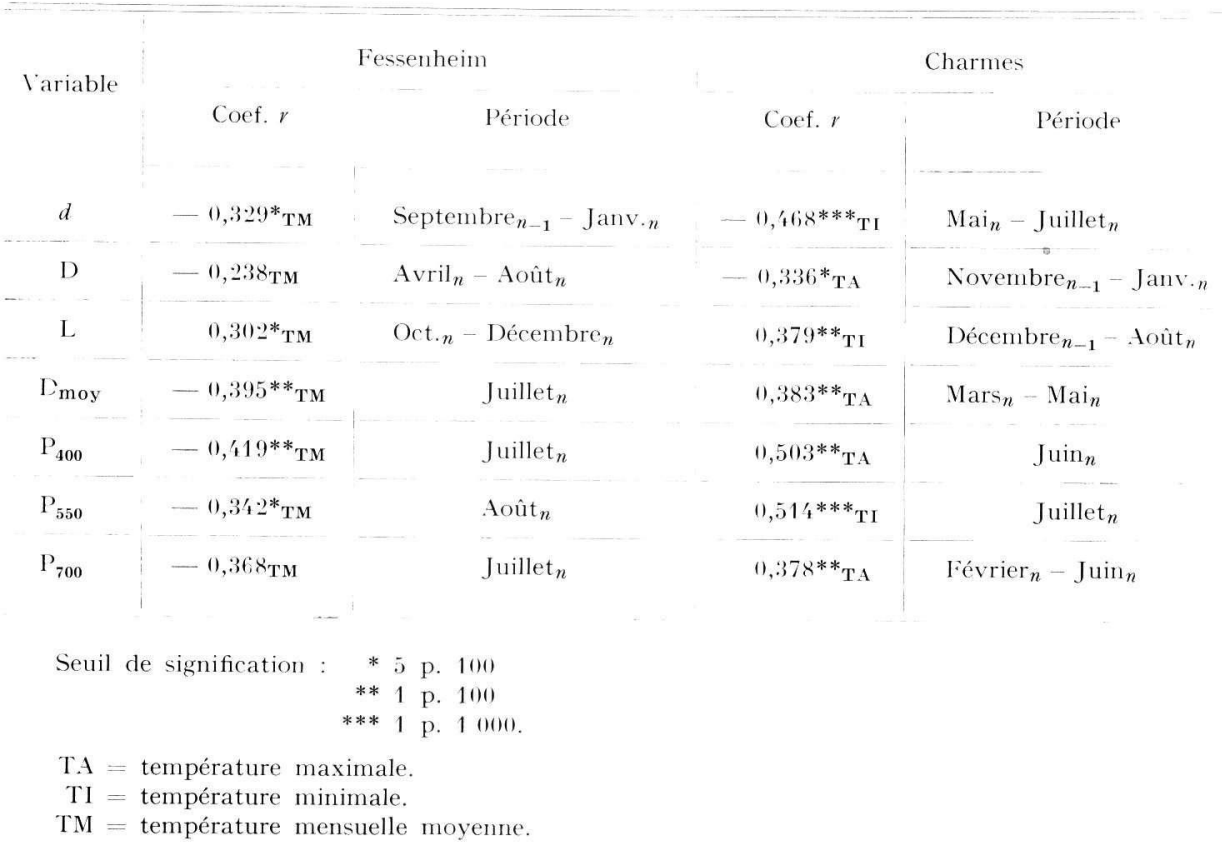

Toutefois, il faut souligner que les deux densités (maximales et minimales) sont liées de façon négative à la température quelle que soit la station, alors que pour la densité moyenne, il y a inversion du signe du coefficient de corrélation suivant le peuplement considéré.

A Fessenheim, le signe du coefficient de corrélation laisserait à penser qu'une température élevée pendant la période de repos végétatif favoriserait, dans une certaine limite, la croissance diamétrale de l'arbre en général, mais donnerait un bois initial de faible densité. Peut-être pourrait-on invoquer, à la reprise de la végétation, une multiplication rapide des cellules cambiales, qui ne se lignifieraient ensuite que très peu!

Si à Fessenheim, les périodes climatiques corrélées aux différentes variables semblent plausibles, à Charmes, elles peuvent susciter l'étonnement : 
- la densité maximale, qui prend sa place en fin d'activité végétative, est ici en liaison avec les températures de novembre à janvier précédant sa formation. Mais il faut remarquer que le coefficient n'atteint qu'un seuil de signification de 5 p. IOO;

- pour la densité minimale (coefficient significatif au seuil de I p. I ooo), nous voyons qu'elle est influencée par les températures de juillet alors que théoriquement, nous sommes dans la phase transitoire entre le bois final et le bois initial. Doit-on en déduire que le bois de printemps subit encore des modifications après sa mise en place?

Travaillant sur Pseudotsuga menziesii, PARker et al. (I974) avaient obtenu des résultats contraires. Seule D offrait une corrélation négative (comme dans cette étude) et significative (comme à Charmes), mais avec un seuil de I p. Ioo et pour des températures allant de mai à aô̂t. Pour les autres données dendrochronologiques, les résultats qu'ils avaient obtenus n'étaient pas concluants.

\section{2. Problème de l'eau}

Nombreux sont les travaux entrepris montrant l'importance de l'eau chez un végétal. Dans cette étude, nous allons essayer de mettre en évidence son importance à partir de données brutes (pluviométrie à Charmes, pluviométrie et nappe à Fessenheim) et à partir de données synthétiques (ETP, RU, BO).

\section{La pluviométrie.}

Quelle que soit la variable, la corrélation arbre-précipitations atteint toujours le seuil de I p. I ooo à Fessenheim, mais c'est à Charmes que le coefficient atteint la valeur la plus élevée avec 0,725 pour $\mathrm{P}_{559}$ (tabl. 8).

Un examen plus attentif fait apparaître des résultats surprenants en ce qui concerne les périodes de précipitations avec lesquelles sont liées les données du bois :

- A Charmes, la densité minimale : bien que le coefficient ne soit pas significatif, il faut le mettre en parallèle avec celui obtenu pour la température. D'autre part, la période qui, au premier abord, pouvait avoir par ses pluies une influence sur $d$ (les mois de mars-avril-mai) offre un coefficient plus faible encore $(-0,065)$.

- A Fessenheim : pour la largeur de cerne, nous trouvons une période curieuse, puisque ce sont les pluies de l'année (août à aô̂t) qui sont corrélées, et ceci au seuil de I p. I ooo avec l'accroissement diamétral. Si nous considérons deux autres périodes se rapprochant de celles trouvées à Charmes, et pour lesquelles on aurait pensé obtenir un coefficient plus élevé, nous trouvons :

$$
\begin{aligned}
\text { pour avril-août : } & 0,36 \text { I } \\
\text { mai-août } & 0,399 \text { (seuil de } 5 \text { p. Ioo). }
\end{aligned}
$$

La densité maximale : elle est corrélée positivement avec les précipitations et ceci peut expliquer les faibles valeurs prises par D en I947, I949, I962 (fig. Io).

Ces résultats sont en contradiction totale avec ceux observés par différents auteurs qui, en général, montraient que la densité maximale était liée de façon négative et très significative avec les précipitations ; c'est le cas chez le Douglas et Abies grandis de la forêt de Haye (H. Polge, I966) et chez Pinus resinosa (Ph. Larsox, I963). 
Liaisons entre les différentes variables

et les précipitations moyennes d'une période considérée

The correlation between the different variables and the average rainfall for a given period

\begin{tabular}{|c|c|c|c|c|}
\hline \multirow{2}{*}{ Variable } & \multicolumn{2}{|r|}{ Fessenheim } & \multicolumn{2}{|c|}{ Charmes } \\
\hline & Coef. $r$ & Période & Coef. $r$ & Période \\
\hline$d$ & $-0,51 \% * * *$ & Déc.n-1 - Mai ${ }_{n}$ & $-0,2990$ & Avril $_{n}$ - Juillet $n$ \\
\hline $\mathrm{D}$ & $0,623 * * *$ & Juin $_{n}$-Octobre $n$ & $0,528 * * *$ & Février $_{n}-\mathrm{Juin}_{n}$ \\
\hline $\mathrm{L}$ & $0,597 * * *$ & Août $_{n-1}-$ Août $_{n}$ & $0,315^{*}$ & Avril $_{n}-$ - $_{\text {oût }} n$ \\
\hline Dmoy & $0,559 * * *$ & Juin $_{n}-$ Août $_{n}$ & $0,61^{\prime}: * * *$ & Juin $_{n}-$ Anôt $_{n}$ \\
\hline$P_{400}$ & $0,180 * * *$ & $\operatorname{Juin}_{n}-$ Août $_{n}$ & $0,6666 * * *$ & $\operatorname{Juin}_{n}-$ toût $_{n}$ \\
\hline $\mathrm{P}_{550}$ & 0,$567 ; * * *$ & Juin $_{n}-$ Juillet $_{n}$ & $0,725 * * *$ & Juin $_{n}-$ Août $_{n}$ \\
\hline$P_{700}$ & $0,548 * * *$ & Mai $_{n}-$ Juillet $_{n}$ & $0,51: 2 * * *$ & Juin $_{n}-$ toût $_{n}$ \\
\hline \multicolumn{2}{|c|}{ Seuil de signification : } & $\begin{array}{rrrl}* & 5 & \text { p. } & 100 \\
* * & 1 & \text { p. } & 100 \\
* * * & 1 & \text { p. } & 1000 .\end{array}$ & & \\
\hline
\end{tabular}
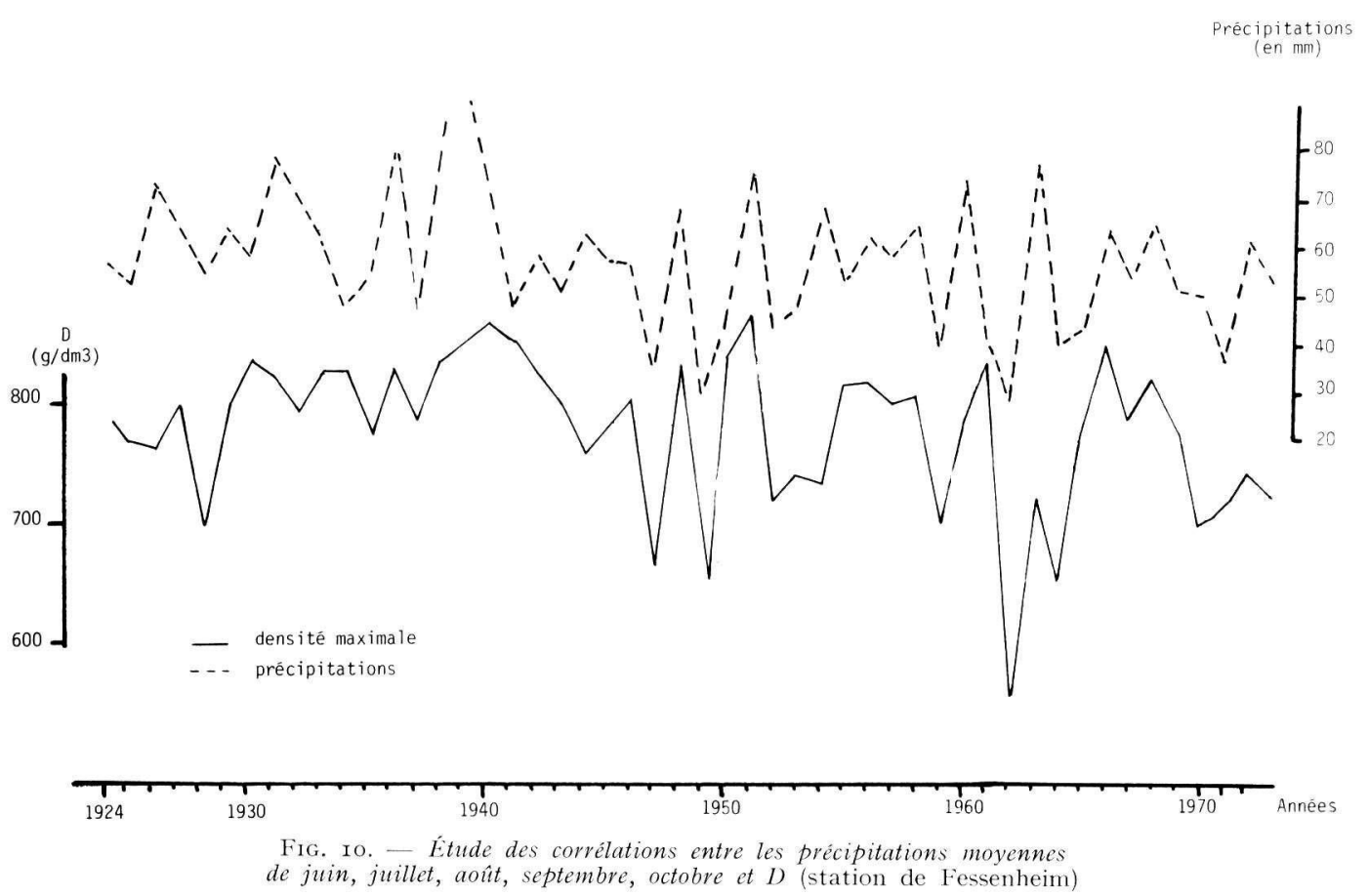

A study of the correlation between the average rainfall for June, July, August, September and October, and the laterood density

(Fessenheim) 
Douglas: le facteur le plus important était la pluviosité des mois d'aoûtseptembre et octobre qui fournissait un coefficient de corrélation négatif et significatif à I p. I ooo.

Abies grandis: ce sont les précipitations d'aô̂t et septembre qui donnaient un coefficient de corrélation négatif et significatif à I p. IOO

Ph. LARson (I963), travaillant sur des plants de 5 ans, montre qu'une période de sécheresse intervenant lors de la formation du bois initial entraîne une diminution de la longueur des aiguilles, une diminution de la teneur en auxine et la formation d'un faux cerne. Si l'approvisionnement en eau redevient normal, l'arbre reproduit un bois initial. Si on freine l'élaboration d'auxine par défoliaison ou passage de l'arbre en jour court, il y a formation de bois final, et ceci de façon irréversible.

LARSON pense que la sécheresse agit de façon directe sur le méristème terminal et qu'une réduction d'eau voit une diminution d'auxine entraînant une diminution de la plasticité de la paroi cellulaire et de la pression de la sève. Ces deux éléments : auxine et turgescence semblent liés directement ou indirectement à la synthèse des constituants de la paroi.

Quoi qu'il en soit, les résultats obtenus dans cette étude sont à mettre en parallèle avec ceux déjà obtenus sur Pinus sylvestris mis en irrigation artificielle par R. Keilier et H. Polge (I968). Ces deux auteurs montrent que si l'apport d'eau cumulé des mois d'août et septembre est supérieur de $80 \mathrm{~mm}$ à celui des mois correspondants de l'année précédente, la densité maximale est de façon très significative plus élevée pour la deuxième année que pour la première

Il faut aussi remarquer la bonne corrélation obtenue avec les trois pourcentages qui dépendent d'une façon générale, pour les deux stations, des précipitations de juin à août, c'est-à-dire de la pluie que reçoit l'arbre lorsque la lumière est la plus forte et qu'il est en pleine activité végétative.

Ainsi, le pin sylvestre pourrait avoir un comportement particulier ris-à-vis de l'eau, mais des expériences complémentaires longues et délicates derraient être entreprises pour en fournir une explication valable.

\section{La nappe souterraine de Fessenheim.}

Devant la profondeur à laquelle se trouve la nappe, la première hypothèse était qu'elle n'avait sûrement aucun effet sur les Pins. D'autre part, l'examen du sol avait révélé que l'enracinement des arbres était de 1'ordre de $\mathrm{I}, 80 \mathrm{~m}$ à $2 \mathrm{~m}$ (BoxNeAu et al., I963). Une alimentation par remontée capillaire pouvait se concevoir difficilement surtout lorsque l'on sait que le sol est constitué presque essentiellement de galets roulés du Rhin. Cependant, une étude de corrélation arbre-nappe (relevés mensuels) nous a fourni des coefficients significatifs pour deux variables (tabl. 9).

A la suite de ces résultats, diverses hypothèses pouvaient être formulées :

- quelle influence avait eu la nappe pendant la période précédant la formation du bois initial?

- existait-il une liaison entre la nappe et les précipitations?

Après de nombreux calculs, le meilleur coefficient a été obtenu lorsque nous corrélions la densité minimale avec les relevés piézométriques de septembre et octobre de l'année précédente : $r=-0,527$, mais le seuil de signification demeure de 5 p. Ioo.

D'autre part, $d$ était corrélé avec les précipitations de décembre à mai, même 
en ne tenant compte que de la période I952-I973 ( $r=-0,546$, seuil de o,oI). Dans quelle mesure la nappe n'était pas sous l'influence de ces pluies? Or la corrélation nous a fourni un coefficient de - o, I 86, done non significatif.

TABI,EAU 9

Étude des corrélations entre la nappe et les données du bois

A study of the correlations between the water table and the variables for the wood

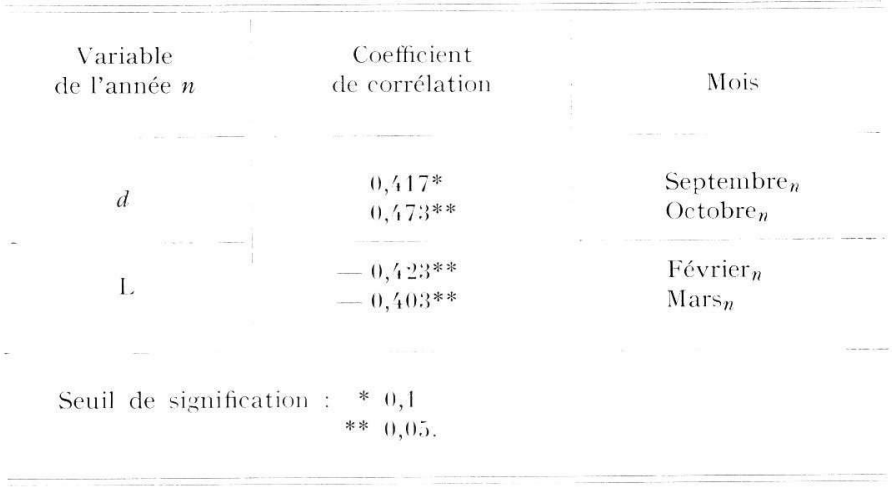

Dans cette seconde éventualité, il faut tenir compte du fait que de 1952 à 1956 , la nappe avait un niveau qui ne pouvait en aucun cas être en liaison avec les précipitations. Démontrer qu'à cette période, la liaison nappe- $d$ existe tendrait à prouver que la nappe influence les arbres et sans intermédiaire des pluies. Lors de la période des travaux nous avons obtenu un coefficient de - 0,949 (significatif à 5 p. IoO pour la corrélation $d$-nappe (septembre-octobre) $n_{-1}$. Doit-on déduire que la nappe influence ces Pins?

D'autres calculs nous ont montré que, sans pouvoir mettre le fait de façon claire en évidence, l'abaissement de cette nappe avait perturbé les arbres.

\section{3. - L'érapotranspiration}

L'E'TP correspond à la hauteur d'eau perdue par un couvert végétal fermé bien alimenté en eau. C'est un facteur physique du climat définissant les besoins théoriques d'un tapis végétal formé d'arbres, arbustes et plantes herbacées. Pour Fessenheim, 1'ETP a été calculée par la formule de Thorntwhaite à partir des températures moyennes mensuelles. Le tableau Io nous permet de constater que les coefficients de corrélation ne sont pas hautement significatifs, et d'autre part qu'ils sont de signe négatif. Seule la largeur de cernes n'est pas liée à l'E'TP de l'une des périodes que nous avons considérées (tabl. 6).

Si nous examinons la formule de Thornthwaite :

$$
\mathrm{E} \text { TTP }=\mathrm{I} 6(\operatorname{IO} t / \mathrm{I})^{a} ; \quad \mathrm{I}=\Sigma_{1_{1}^{2}} i
$$

où $i=(t / 5) \mathrm{I}, 5 \mathrm{I} 4$;

$a=0,0 \mathrm{I} 6 \mathrm{I}+0,5\left(t\right.$ en ${ }^{\circ} \mathrm{C}$, ETP en mm $)$, 
FiTP dépend essentiellement de la température pour une région donnée; c'est peut-être la raison pour laquelle nous avons une analogie dans les signes des coefficients de corrélation entre ce tableau io et le 8 .

TABLEAU IO

Corrélations entre les différentes variables et l'évapotranspiration

The correlation between the different variables and evaporation

\begin{tabular}{|c|c|c|}
\hline Variable & Coefficient $r$ & Période \\
\hline$d$ & $-0,300 *$ & Mars $_{n}-$ Août $_{n}$ \\
\hline 1) & $-0,359^{*}$ & Août $_{n}$ \\
\hline $\mathrm{L}$ & $-0,259$ & Avril $_{n}-$ Août $_{n}$ \\
\hline$D_{\operatorname{moy}}$ & $-0,403 * *$ & Avril $_{n}-$ Août $_{n}$ \\
\hline$P_{400}$ & $-0,396^{*}$ & Juillet $_{n}$ \\
\hline$\Gamma_{550}^{\prime}$ & $-0,390 * *$ & Avril $_{n}-$ Août $_{n}$ \\
\hline$P_{700}$ & $-11,413^{* *}$ & Avril $_{n}-$ Août $_{n}$ \\
\hline Seuils de & $\begin{array}{rlll}* & 5 & \text { p. } & 100 \\
* * & 1 & \text { p. } & 100 .\end{array}$ & \\
\hline
\end{tabular}

Les valeurs de l'E'TP doivent être en réalité manipulées avec prudence, car elles ont été formulées pour un couvert végétal complet dans l'hypothèse où la transpiration n'était pas limitée par la disponibilité d'eau (ce qui n'est pas le cas à Fessenheim).

Il aurait donc été préférable d'employer l'évapotranspiration réelle (ETR) ou une formule comparant l'eau disponible pour la plante (réserve du sol + précipitations) à l'évapotranspiration potentielle par exemple :

$$
\frac{\mathrm{R}_{(n-1)}+\mathrm{P}_{n}}{\mathrm{E}^{\prime} \mathrm{TP} P_{n}}(2) \quad \text { (G. AUssenac, I973) }
$$

$\mathrm{R}_{(n-1)}=$ réserve en eau à la fin du mois $(n-\mathrm{I})$,

$\mathrm{P}_{n}=$ précipitations du mois $n$,

$\mathrm{ETP}_{n}=$ évapotranspiration potentielle du mois $n$.

Bien entendu, plus ce rapport (2) se rapproche de I, plus l'alimentation en eau de l'arbre est optimale.

\section{4. - Les réserves utiles}

A partir de la donnée théorique qu'est 1'E'TP, il a été calculé à la fin de chaque mois la réserve utile des trois sols ayant respectivement :

$$
\begin{aligned}
& \mathrm{RU}_{1}=35 \mathrm{~mm} \\
& \mathrm{RU}_{2}=70 \mathrm{~mm} \\
& \mathrm{RU}_{3}=140 \mathrm{~mm} .
\end{aligned}
$$


Le graphique de la figure I m montre les différences que l'on peut observer d'un sol à l'autre. Le sol de réserve utile $35 \mathrm{~mm}$ atteint dès le mois de mai le point de flétrissement $(\mathrm{RU}=0)$ qui demeure ainsi jusqu'en août, soit 3 mois. Pour le sol de réserve $\mathrm{I}$ fo $\mathrm{mm}$, le point de flétrissement est atteint en juillet et ne demeure qu'un mois ; cette remarque, faite pour l'année 1952 est, nous l'avons vérifié, valable pour la moyenne des jo années étudiées.

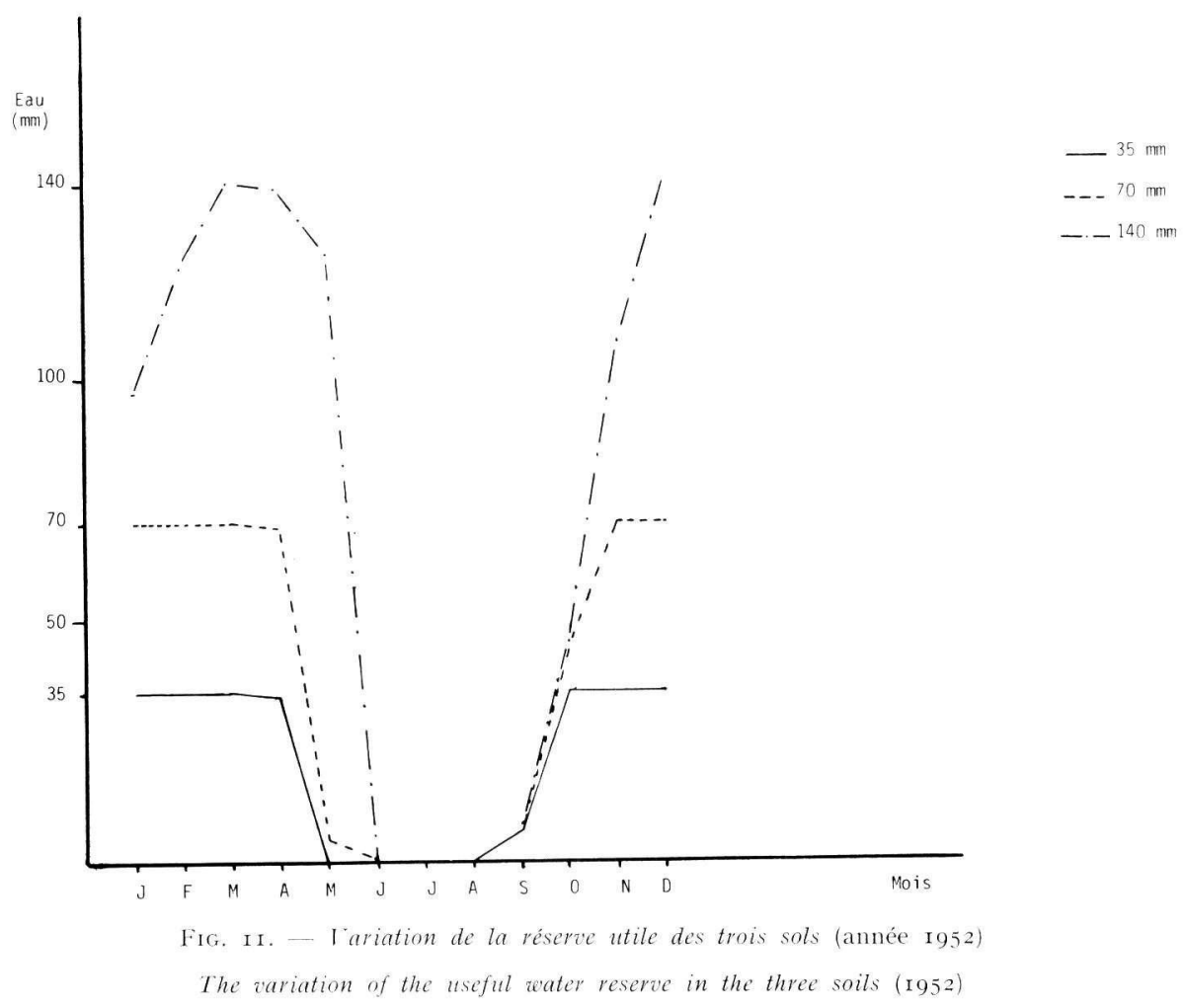

Ce sont les corrélations arbres- $\mathrm{RU}_{3}$, dont nous dressons ici le tableau (tabl. I I) qui nous ont donné les meilleurs résultats :

Si nous regardons le signe de ces coefficients, nous aboutissons aux mêmes conclusions que pour les précipitations.

Tout apport d'eau à l'arbre est lié positivement aux données dendrochronologiques, sauf pour $d$ où l'apport d'eau croissant fait diminuer sa valeur.

Cependant, si nous regardons les périodes liées aux différentes données, nous constatons qu'elles sont souvent inattendues; c'est le cas de $\mathrm{P}_{700}$; nous pensons que ce n'est pas cette période qui est la plus favorable, mais simplement que $\mathrm{P}_{700}$ est d'autant plus élevé que RU est importante. D'autre part, les calculs sont faussés dans la mesure où les valeurs oscillent entre o-I4O. Une nouvelle fois se pose le problème des limites; certaines années pourront connaitre un accroissement double de celui de l'année passée, sans pour autant que la valeur RU soit multipliée par deux. 
TABLEAU II

Corrélations entre les différentes variables et la $\mathrm{RU}_{\mathbf{3}}$

The correlation between the different variables and $\mathrm{RU}_{3}$

\begin{tabular}{|c|c|c|}
\hline $\begin{array}{c}\text { Variables } \\
\text { de l'année } n\end{array}$ & Coefficient & Période \\
\hline$d$ & $-0,288^{*}$ & Avril $_{n}-\mathrm{Juin}_{n}$ \\
\hline D) & $0,3 \div 1 *$ & $\mathrm{Janv}_{n}-\operatorname{Mars}_{n}$ \\
\hline $\mathrm{L}$ & $0,550 * * *$ & $\operatorname{Mars}_{n}-$ Août $_{n}$ \\
\hline $\mathrm{D}_{\operatorname{mos}}$ & $0,346 *$ & Sept. $n_{-1}-$ Déc. $n_{-1}$ \\
\hline$\% d \geqslant 400$ & \multicolumn{2}{|c|}{ Pas de corrélation significative } \\
\hline$\% d \geqslant 550$ & $0,308 *$ & Août $_{n_{-1}}-$ Déc. $_{n_{-1}}$ \\
\hline$\% d \geqslant 700$ & $0,350^{*}$ & Sept. $n_{-1}-$ Déc. $n_{-1}$ \\
\hline Seuil de signification : & $\begin{array}{rl}* & * 0,05 \\
* & * 0,01 \\
* * * 0,001\end{array}$ & \\
\hline
\end{tabular}

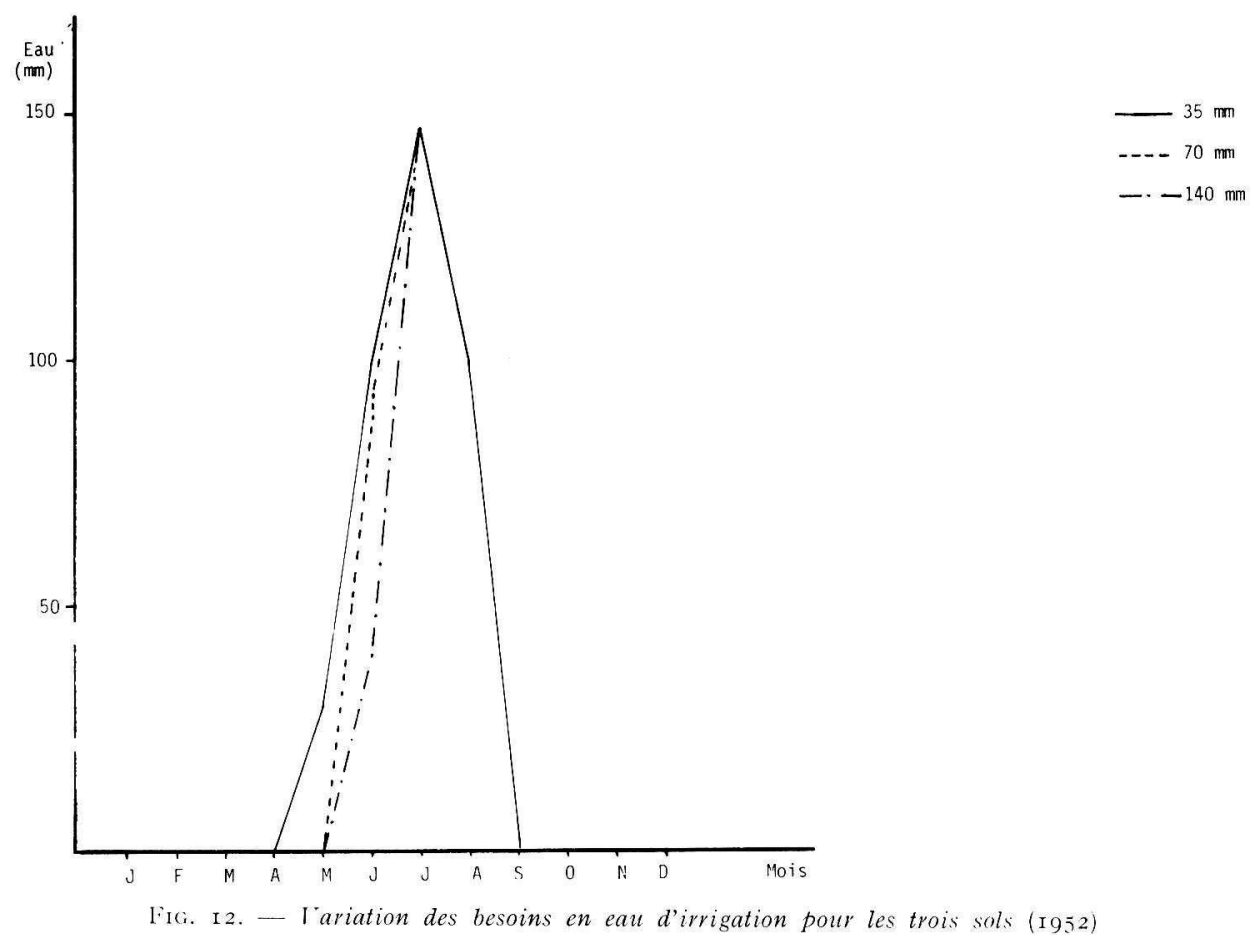

The tariation of irrigation requirements in the 3 soils (1952) 


\section{5. - Les besoins en ean}

C'est ici une valeur théorique donnée pour les trois sols, théorique car les pins ont connu des besoins en eau, mais n'ont jamais été arrosés. Les besoins en eau vont donc dans ce cas présent traduire la sécheresse subie par les arbres, sécheresse d'autant plus précoce que le sol avait une réserve utile faible. Dès que les besoins en eau se font sentir, on a atteint, quel que soit le sol, le point de flétrissement ; à partir de ce moment, l'absorption racinaire est donc nulle. Malgré la différence de réserve en eau, ces besoins se font sentir entre avril et mai (fig. I2) ; seule la quantité d'eau absorbée par les arbres avant cette période varie.

TABLEAU I 2

Étude des corrélations entre les différentes variables

et les besoins en eau de complément

1 study of the corvelation between the different variables and the additional water requirements

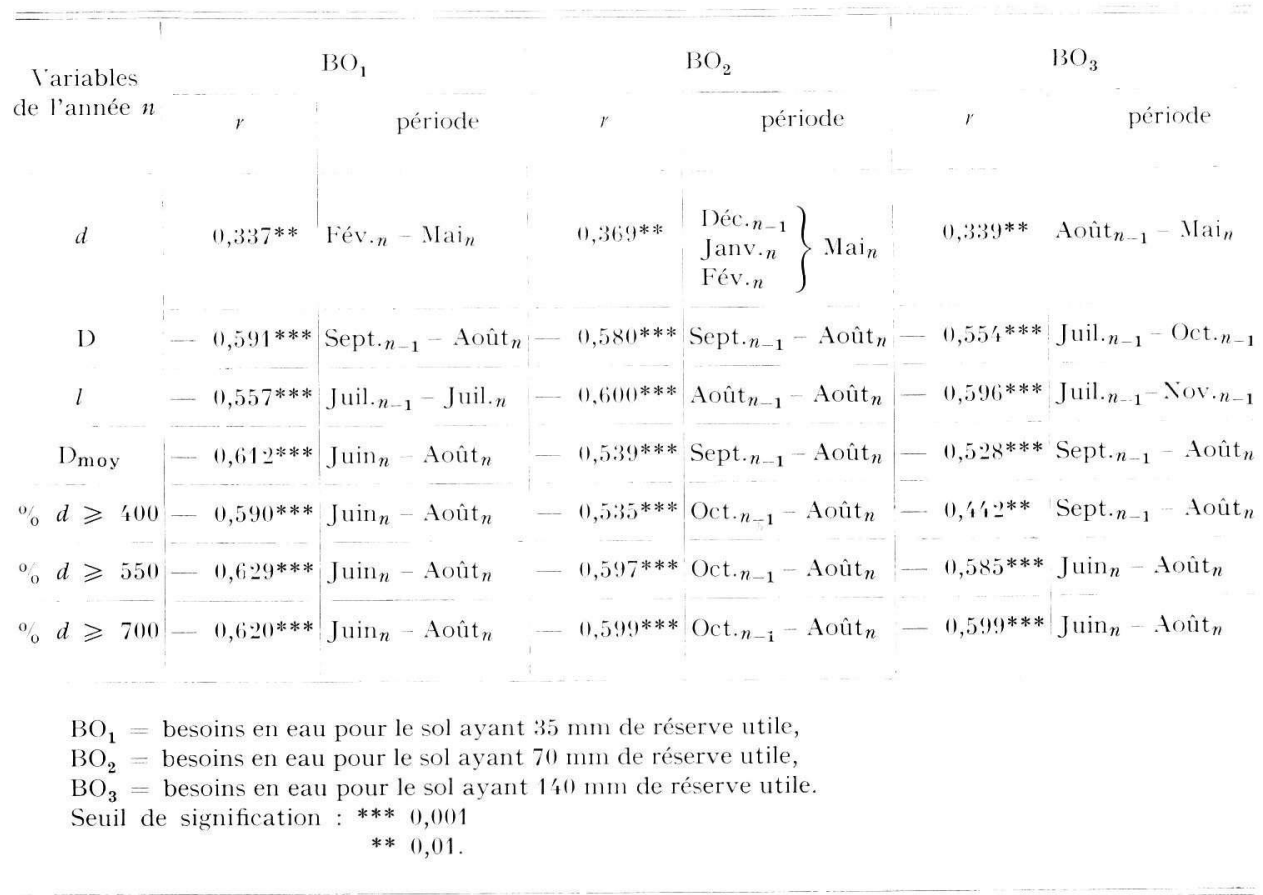

Il est difficile de tenir compte des périodes du tableau I2 car elles sont longues et donc sensiblement de même valeur. Cependant, à Fessenheim, il faut remarquer que plus la période de juin à août est sèche, plus $\mathrm{P}_{550}$ et $\mathrm{P}_{700}$ sont faibles. 


\title{
Conclusion
}

Cette étude nous a permis de mettre en évidence un fait concernant la méthode d'étude de la dendrochronologie, l'autre concernant l'arbre et le climat.

L'utilisation des coefficients de sensibilité et d'autocorrélation ne présente pas un intérêt évident. Nous leur préférerons l'emploi des années caractéristiques, méthode simple donnant des résultats permettant des conclusions cohérentes.

L'autre remarque concerne la relation arbre-climat et aussi sol.

A Fessenheim, le climat sec et le sol filtrant peuvent expliquer que ce peuplement soit plus sensible aux précipitations qu'à la température, car la pluie semble être le facteur limitant.

A Charmes, au contraire, l'humidité atmosphérique est plus importante; les arbres poussent aussi sur des sols à nappes perchées temporaires (présence de pseudogley) et jouissent pratiquement toute l'année d'une bonne alimentation en eau, d'une température plus régulière et sont dans l'ensemble moins soumis à des variations brutales du climat.

Par ailleurs, pour un couvert forestier tel que celui de Fessenheim, c'est avec prudence que l'on doit faire usage des variables climatiques synthétiques car le peuplement ne répond pas aux conditions d'utilisation de l'E'TP par exemple. D'autre part, les valeurs des précipitations utilisées dans cette étude sont des valeurs globales qui ne tiennent pas compte du fait que dans la Hardt au climat continental, beaucoup se produisent sous forme d'orage ; une grande partie de l'eau pourrait alors être non utilisée et disparaître sous forme de drainage.

Einfin, la dernière remarque est sûrement de loin la plus importante ; elle concerne la densité maximale qui est liée de façon positive aux précipitations d'une période bien définie (tabl. 8). Ce résultat est contraire à ceux qu'on peut lire dans la littérature (les études déjà faites concernaient d'autres résineux).

Une explication valable de cette opposition ne pourrait être proposée que si l'on connaissait exactement la période de croissance en circonférence des deux espèces concernées et si l'on pouvait, en milieu contrôlé, étudier sur modèles réduits leurs besoins en eau.

Ręu pour publication en janvier 1976 .

\author{
SUMMARY
}

PROBLEMS IN CROSS-DATING NORWAY PINES

ANI) THE INFLUENCL OF CLIMATE ON THE STRUCTURE OF ANNUAI, INCREMENT

()ur research concerned itself with the study of two groups of 25 pines (Pinus sylvestris), one in Charmes (Vosges), the other in Fessenheim (Lower Rhine region).

In the first part of our study, our purpose was to find a method which characterizes a group. We have referred to the sensitivity coefficient, the " auto-correlation " coefficient, the cross-dating cocfficient, and the "characteristic years" method.

The latter, a simple method, proved to be the most efficient, as was the case for the dendrochronological study. 
The second part concerned itself with the influence of weather on these trees. We have thus shown that latewood density was positively dependant upon rain.

We have taken into account the rain fall for July, August and September of the present year for the group in Charmes and the rainfall for April, May and June of the same year for the group in Fessenheim.

\section{ZUSAMMENFASSUNG}

PROBLEME DER JAHRRINGDATILIERUNG UND DER EINFIUSS DES KLIMAS AUF DIE STRUKTUR DER JAHRRINGE BEI DER KIEFER (PINUS SYLVESTRIS)

Unsere Arbeit bezog sich auf das Studium von zwei Kiefernbestände (Pimus sylvestris), der eine in Charmes (Vosges), der andere in Fessenheim (Bas-Rhin).

Im ersten Teil unserer Arbeit haben wir eine Methode gesucht die den Bestand karaktesiert. Die Methode der karateristischen Jahrringe, eine einfache Methode, ist die beweiskräftigste für dendrochronologische Studien.

Im zweiten Teil, haben wir den Einfluss des Klimas auf diese Baüme studiert.

So haben wir bewiesen, dass das spezifische Gewicht des Spätsholzes direkten Weise von den Niederschlagen abhängt.

- Für Charmes von den Niederschlägen der Monate: Juli-August, September und Oktober,

- Für Fessenheim von den Niederschlägen der Monate Februar. März, April und Juni des laufenden Jahres.

\section{RÉFÉRENCES BIBIIOGRAPHIQUES}

Aussenac (i., I973. Clinat, microclimat et production ligneuse. Innales des Sciences forestieres, $\mathbf{3 0}$ (3), 239-258.

Becker M., ig7I. Litude de la relation sol-végétation en condition a'hydromorphie, dans la forêt de la plaine Lorraine. Thèse de Doctorat d'État, Sciences naturelles, Université de Nancy I.

Bonneau M., Henrich J. C., Levy G., I963. Étude d'un bilan hydrique "les îles du Rhin en Alsace. Revue forestiere Française, $\mathrm{n}^{\mathrm{O}} 4$.

Brethes A., I973. Communication personnelle.

Larson Ph., 1963. The indirect effect of drought on tracheid diameter in Red Pine. Forest Science, 9 (I), 53-62.

Munaut A. V., 1966. Recherches dendrochronologiques sur Pinus silvestris. I. Étude de 45 pins sylvestres récents originaires de Belgique. A gricultura (Louvain), 14 (2), 193-232.

Parker M. L., Henoch W. E. S., I97I. The use of Engelmann Spruce latewood density for dendrochronological purpose. Canadian Journal of Forest Research, 2.

Parker M. L., Heger L., Kennedy R. W., I974. X-ray densitometry : a technique and an example of applicata. Wood Science, $\gamma$ (2), r40-I 48 .

Polge H., I966. Établissement des courbes de variation de la densité du bois par exploration densitométrique d'échantillons prélevés à la tarière sur des arbres vivants. Applications dans les domaines technologique et physiologique. Thèse Docteur ès-Sciences Appliquées, Faculté des Sciences de l'Université de Nancy, 27 juin.

Polge H., Keller R., I968. Influence de l'approvisionnement en eau sur la structure interne des accroissements annuels. Annales des Sciences forestives, 25 (3), I25-133. 